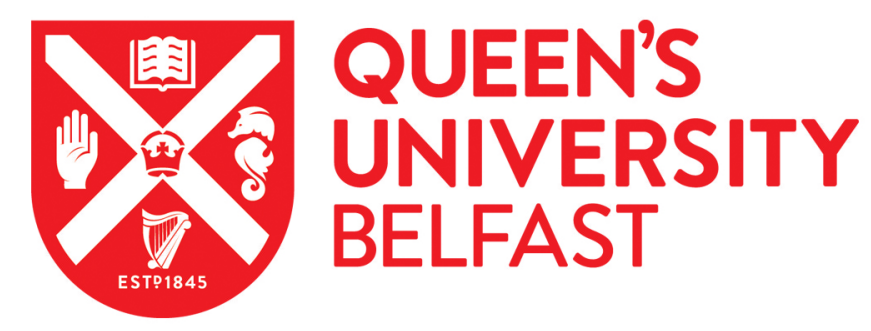

\title{
Radiation-hydrodynamic simulations of thermally-driven disc winds in X-ray binaries: A direct comparison to GRO J1655-40
}

Higginbottom, N., Knigge, C., Long, K. S., Matthews, J. H., Sim, S. A., \& Hewitt, H. A. (2018). Radiationhydrodynamic simulations of thermally-driven disc winds in X-ray binaries: A direct comparison to GRO J165540. Monthly Notices of the Royal Astronomical Society, Advance Access, 1-13. https://doi.org/10.1093/mnras/sty1599

Published in:

Monthly Notices of the Royal Astronomical Society, Advance Access

Document Version:

Peer reviewed version

Queen's University Belfast - Research Portal:

Link to publication record in Queen's University Belfast Research Portal

Publisher rights

(C) 2018 The Author(s) Published by Oxford University Press on behalf of the Royal Astronomical Society. This work is made available online in accordance with the publisher's policies. Please refer to any applicable terms of use of the publisher.

\section{General rights}

Copyright for the publications made accessible via the Queen's University Belfast Research Portal is retained by the author(s) and / or other copyright owners and it is a condition of accessing these publications that users recognise and abide by the legal requirements associated with these rights.

Take down policy

The Research Portal is Queen's institutional repository that provides access to Queen's research output. Every effort has been made to ensure that content in the Research Portal does not infringe any person's rights, or applicable UK laws. If you discover content in the Research Portal that you believe breaches copyright or violates any law, please contact openaccess@qub.ac.uk. 


\title{
Radiation-hydrodynamic simulations of thermally-driven disc winds in X-ray binaries: A direct comparison to GRO J1655-40
}

\author{
Nick Higginbottom, ${ }^{1 \star}$ Christian Knigge ${ }^{1}$, Knox S. Long ${ }^{2,3}$, James H. Matthews ${ }^{4}$, \\ Stuart A. Sim ${ }^{5}$ and Henrietta A. Hewitt ${ }^{5}$. \\ ${ }^{1}$ School of Physics and Astronomy, University of Southampton, Highfield, Southampton, SO17 1BJ, UK \\ ${ }^{2}$ Space Telescope Science Institute, 3700 San Martin Drive, Baltimore, MD, 21218, USA \\ ${ }^{3}$ Eureka Scientific Inc., 2542 Delmar Avenue, Suite 100, Oakland, CA, 94602-3017, USA \\ ${ }^{4}$ University of Oxford, Astrophysics, Keble Road, Oxford OX1 3RH, UK \\ ${ }^{5}$ School of Mathematics and Physics, Queen's University Belfast, University Road, Belfast BTr 1NN, UK
}

Accepted XXX. Received YYY; in original form ZZZ

\begin{abstract}
Essentially all low-mass X-ray binaries (LMXBs) in the soft state appear to drive powerful equatorial disc winds. A simple mechanism for driving such outflows involves $\mathrm{X}$-ray heating of the top of the disc atmosphere to the Compton temperature. Beyond the Compton radius, the thermal speed exceeds the escape velocity, and mass loss is inevitable. Here, we present the first coupled radiation-hydrodynamic simulation of such thermally-driven disc winds. The main advance over previous modelling efforts is that the frequency-dependent attenuation of the irradiating SED is taken into account. We can therefore relax the approximation that the wind is optically thin throughout which is unlikely to hold in the crucial acceleration zone of the flow. The main remaining limitations of our simulations are connected to our treatment of optically thick regions. Adopting parameters representative of the wind-driving LMXB GRO J1655-40, our radiation-hydrodynamic model yields a mass-loss rate that is $\simeq 5 \times$ lower than that suggested by pure hydrodynamic, optically thin models. This outflow rate still represents more than twice the accretion rate and agrees well with the mass-loss rate inferred from Chandra/HETG observations of GRO J1655-40 at a time when the system had a similar luminosity to that adopted in our simulations. The Fe XXV and Fe XXVI Lyman $\alpha$ absorption line profiles observed in this state are slightly stronger than those predicted by our simulations but the qualitative agreement between observed and simulated outflow properties means that thermal driving is a viable mechanism for powering the disc winds seen in soft-state LMXBs.
\end{abstract}

Key words: Accretion discs - hydrodynamics - methods:numerical - stars:winds X-rays:binaries

\section{INTRODUCTION}

Outflowing gas is seen in a vast range of accreting systems from cataclysmic variables (e.g. Cordova \& Mason 1982; Körding et al. 2011) to active galactic nuclei (AGN) (e.g. Blandford \& Payne 1982; Weymann et al. 1991; Turner \& Miller 2009). These outflows often have very high velocities and appear to transport a great deal of material from the accreting object out to its environment. There are, broadly speaking, two classes of outflows: highly collimated fast po-

^ E-mail: nick_higginbottom@fastmail.fm lar jets (e.g. Zensus 1997; Mirabel \& Rodríguez 1999; Remillard \& McClintock 2006) and weakly collimated disc winds (e.g. Konigl \& Kartje 1994; Knigge et al. 1997; Díaz Trigo \& Boirin 2013). In general, the former are easier to observe because they can remain distinct over great distances. The latter tend to reveal themselves only via blue shifted absorption features, but have the potential for a greater impact on their surroundings, because they transport much more mass and momentum (Fabian 2012).

This effect on their environment is one reason for the astrophysical importance of disc winds. Another is that the removal of mass and angular momentum from the disc might 
fundamentally change the accretion process itself (Shields et al. 1986). In addition, if such outflows are ubiquitous, then most observations of disc-accreting systems may contain signatures of these disc winds and need to be interpreted with this in mind.

Low-mass X-ray binaries (LMXBs) are excellent laboratories for studying accretion physics. First, they are much closer than the more dramatic AGN, and are therefore somewhat easier to observe. Second, and more importantly, they exhibit dramatic "state changes" over fairly short timescales of days-weeks (Belloni 2010). These shifts from a low-luminosity spectrally hard (low-hard) state to a highluminosity spectrally soft (high-soft) state are usually attributed to changes in the accretion disc (Ponti et al. 2012). LMXBs spend most of their time in the low-hard state (Belloni 2010), in which the inner part of the geometrically thin accretion disc is truncated far away from the central object. A hot, geometrically thick and optically thin accretion flow (aka "corona") takes over from the disc at smaller radii and produces most of the hard X-ray emission that dominates in this state. During a transition to the high-soft state the inner disc edge is thought to move inward to smaller radii, thus increasing in luminosity and producing a softer overall spectrum.

Clear observational evidence for strong disc winds has so far been seen in 8 LMXBs (Díaz Trigo \& Boirin 2016). In all cases, these systems were in the disc-dominated high-soft state. Moreover, 7 of the 8 sources are known to exhibit dips in their light curves - clear evidence that they are viewed edge on (Frank et al. 1987). Taken together, this suggests that the outflows themselves are equatorial and associated with the accretion disc (Ponti et al. 2012). The association between disc winds and high-soft states might also point to a more fundamental role for these outflows in triggering state transitions. For example, if the wind transports enough mass away from the accretion disc, this might force the system to change back to the low-hard state (Shields et al. 1986).

The driving mechanism for the disc winds seen in LMXBs is not clear. There are three main possibilities: radiation driving (e.g. Icke 1980; Shlosman et al. 1985; Shlosman \& Vitello 1993; Proga \& Kallman 2002), magneto-centrifugal driving (e.g. Blandford \& Payne 1982; Emmering et al. 1992; Miller et al. 2006; Chakravorty et al. 2016) and thermal driving (Begelman et al. 1983; Woods et al. 1996; Luketic et al. 2010).

Radiation driving acting alone is probably the least promising of these candidates. Even in the high-soft state, disc luminosities tend to be below the Eddington limit, so continuum driving alone is usually not sufficient. Line driving is also problematic because the X-ray irradiated gas in these systems is too highly ionized (Díaz Trigo \& Boirin 2016).

Magneto-centrifugal driving, where gas is loaded onto magnetic field lines threading the accretion disc and accelerated outwards along the lines like 'beads-on-a-wire' offers a potential mechanism for winds that appear to arise very close in to the central object. This has been suggested as the driving mechanism for a very dense and fast outflow seen in GROJ1655-40 (Stone \& Norman 1992; Miller et al. 2006, 2008; Kallman et al. 2009, but also see Netzer 2006; Uttley \& Klein-Wolt 2015; Shidatsu et al. 2016 and Section 2).

Thermal driving is a very simple and attractive mecha- nism for most of the disc winds seen in LMXBs. A thermallydriven outflow will arise naturally when the surface of the outer accretion disc is heated to the Compton temperature by X-rays produced in the inner disc and/or the corona. The location in the disc where the thermal velocity at the Compton temperature equals the local escape velocity is referred to as the Compton radius. Beyond this point, the thermal velocity in the upper disc atmosphere exceeds the escape velocity, so material will expand and escape in an out-flowing disc wind.

In practice, the radius at which thermal driving becomes effective is about $10 \%$ of the Compton radius (Begelman et al. 1983). For very luminous sources, where the radiation pressure on electrons reduces the effective gravity significantly, the critical radius can be even smaller (Proga \& Kallman 2002). Nevertheless, thermal winds preferentially arise at rather large distances from the central object. This is consistent with the observation that outflows are preferentially observed in long-period systems with large accretion discs (Díaz Trigo \& Boirin 2016).

Given that disc winds may play a crucial role in triggering state transitions, as well as their apparent ubiquity in disc-dominated states (Ponti et al. 2012), it is important to understand the nature of these outflows. Moreover, since some thermally driven mass loss from the outer disc must occur in many systems, it is natural to explore this mechanism carefully first. Here, we therefore continue a project to numerically simulate thermally driven disc winds in LMXBs and present a direct comparison of our most realistic simulation to observations.

A key assumption made in all simulations of thermally driven disc winds to date is that the outflow is completely optically thin throughout. This matters. The strength and character of these outflows depends strongly on the thermal stability of material in the disc atmosphere (Higginbottom \& Proga 2015; Higginbottom et al. 2017, hereafter HP15 and H17 respectively), and this in turn depends strongly on the shape of the irradiating SED (Dyda et al. 2017). In an optically thin outflow irradiated by a central source, all parts of the wind see exactly the same irradiating spectrum, with a normalization set simply by geometric dilution. However, if the outflow is self-shielding, such approximations break down. To see this, consider a parcel of gas near the wind acceleration zone at some radius $R_{1}$ from the central source. If the irradiating SED reaching this parcel is strongly attenuated by absorption and/or scattering in the wind at radii $R<R_{1}$, the thermal state of this parcel can change dramatically, even to the point where it may no longer be significantly accelerated at all.

Given the sensitivity of thermally driven disc winds to self-shielding effects, it is clearly important to relax the $\tau<<1$ approximation. This is the goal of the present study. Of course, relaxing the "no-self-shielding" approximation turns a pure hydrodynamics problem into a radiationhydrodynamics (RHD) one. As described in more detail below, we tackle this by coupling the well-known zEus hydrodynamics code (Stone \& Norman 1992, extended by Proga et al. 2000), with our own state-of-the-art ionization and radiative transfer code PYTHON (Long \& Knigge 2002, extended by Higginbottom et al. 2013 and Matthews et al. 2015).

Our RHD simulations of thermally driven disc winds 
improve on existing work in two key areas. First, the attenuation of the irradiating SED by the opacity in the outflow is fully taken into account. Second, the resulting spatially and spectrally varying radiation field is used to estimate the thermal and ionization state of the wind. As we shall see, self-shielding is not a second order effect. The local radiation field in key parts of the wind can differ drastically by orders of magnitude at frequencies around the Hydrogen photo-ionization edge - from the unattenuated X-ray spectrum produced by the central source. Perhaps unsurprisingly, this has a significant effect on the resulting outflow. As we shall see, the properties of the thermally driven wind seen in our RHD simulation are a surprisingly good match to those inferred observationally for the well-known wind-driving LMXB GRO J1655-40 in its soft-intermediate state.

\section{GRO J1655-40 AS A BENCHMARK}

We adopt system parameters designed to roughly describe the accreting black hole system GRO J1655-40, a well-known wind-driving LMXB. This choice allows a direct comparison with previous work (Luketic et al. 2010, HP15 and H17) and provides us with high-quality observational data against which we can test our model.

Specifically, we assume a central object mass of $7 M_{\odot}$, a luminosity of $3.3 \times 10^{37} \mathrm{ergs} \mathrm{s}^{-1}\left(\simeq 0.04 \mathrm{~L}_{\mathrm{Edd}}\right)$ and the same bremsstrahlung-type SED used by HP15 and H17,

$L_{v}=K v \exp \left(-h v / k_{B} T_{x}\right)$.

Given a typical accretion efficiency of $8.3 \%$, this luminosity implies a mass accretion rate of $\dot{\mathrm{M}}_{\mathrm{acc}}=4.4 \times 10^{17} \mathrm{~g} \mathrm{~s}^{-1}$. We use the same X-ray temperature as in $\mathrm{H} 17, T_{x}=5.6 \times 10^{7} \mathrm{~K}$, which corresponds to a Compton temperature of $T_{I C}=1.4 \times$ $10^{7} \mathrm{~K}$.

It is crucial to note that these parameters are not appropriate for modelling the April 2005 Chandra observations of GRO J1655-40 described by Miller et al. (2006, also see Miller et al. 2008 and Kallman et al. 2009), which were the basis for their claim of a magnetically driven outflow from the system. The original estimate of $L \simeq 0.04 L_{E d d}$ for this data set has been challenged by Ponti et al. (2012, on the basis of the X-ray continuum), by Uttley \& Klein-Wolt (2015, on the basis of timing properties) and by Neilsen et al. (2016) and Shidatsu et al. (2016, on the basis of the OIR to X-ray SED). Several of these recent studies suggest that, at the time of these observations, the system was in an unusual "hypersoft" state characterized by $L>0.1 L_{E d d}$, and indeed most likely $L \gtrsim L_{E d d}$ (Uttley \& Klein-Wolt 2015; Shidatsu et al. 2016; Done et al. 2018). This hypersoft state is very similar to that seen in the high-mass X-ray binary Cyg X-3, also thought to be accreting at or above the Eddington limit (Uttley \& Klein-Wolt 2015). It remains to be seen whether the need for a magnetic contribution to the wind-driving mechanism in the hypersoft state survives this revision. In any case, data obtained in the hypersoft state clearly cannot be used to test models of thermally driven disc winds in the normal soft state.

Fortunately, Chandra obtained another observation of GRO J1655-40 just 20 days earlier, in March 2005 (Miller et al. 2008; Neilsen \& Homan 2012). This observation took place close to the peak of the outburst and near the end of the hard-to-soft state transition. We will refer to the state of the system at this time as a "soft-intermediate state". Ponti et al. (2012) estimate $L \simeq 0.04 L_{E d d}$ for this state from their modelling of the Chandra $+R X T E$ X-ray continuum. As shown in Figure 1, the soft-intermediate state spectrum exhibits significantly fewer and weaker absorption lines than the hypersoft spectrum. In Section 5.3, we will use this March 2005 spectrum as a testbed for our RHD simulations.

\section{COMPUTATIONAL METHOD}

\subsection{Overview}

Our RHD simulations use an operator-splitting technique to couple zEUs to our Monte Carlo ionization and radiative transfer code PYTHON. This technique permits a complete treatment of radiative transfer (RT) between the source of radiation and each cell in the model. PYTHON calculates the ionization state of the plasma using the local radiation field, which ensures that energy deposition is calculated accurately, even for highly frequency dependent processes like photo-ionization. This represents a significant advance over previous efforts to model such disk winds, which have either assumed optically thin conditions or grey attenuation.

In the course of an RHD calculation, we continuously cycle between hydrodynamic (HD) steps carried out by zEUS and ionization/radiative transfer (IRT) steps carried out by PYTHON, with information about the state of the outflow being passed back and forth between the two codes. In each IRT step, PYTHON takes the latest snapshot of the outflow (i.e. the density, temperature and velocity field across the simulation grid) and injects a population of $N=10^{7}$ "photons" (strictly energy packets) that correctly represents the source SED. The code then follows the passage of these photons through the outflow, keeping track of the energy transfer between the radiation field and the plasma. This information is then used to estimate the ionization state of each grid cell, as well as its heating and cooling rate. In practice, we actually carry out two iterations of this process during each call to PYTHON, in order to ensure that our estimate of the ionization state is converged. Test calculations confirm that two iterations are sufficient to achieve this.

Internally, the version of zEUS used here represents the heating and cooling rates via modified versions of the approximate analytic formulae provided by Blondin (1994). This is similar to the approach adopted by Luketic et al. (2010), HP15 and H17. Our versions of these formulae are given in Equations 2-8 below and discussed in the accompanying text.

The standard normalization of these analytic approximations was calibrated for a specific incident SED. However, since our calculations account for attenuation effects, each cell in our simulation can see a different SED. We therefore re-calibrate each heating/cooling term in the analytic rates for each grid cell after each IRT step by a multiplicative normalization factor - the $K$-terms in equations 2-8. Since PYTHON is typically only called every $t_{H D \text {, max }}$ simulation seconds (see below), we can then use these recalibrated formulae to continuously (if approximately) update the heating/cooling rates between calls to PYTHON. After each call to 


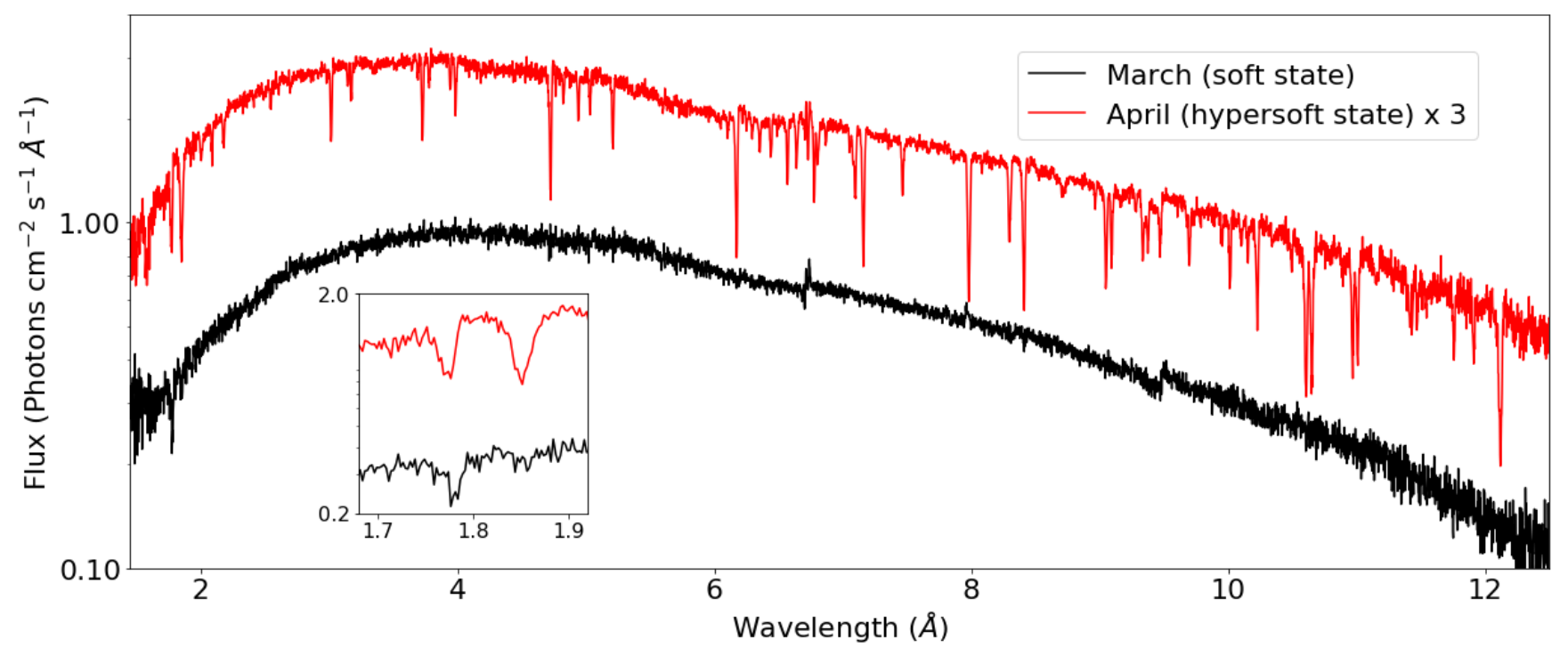

Figure 1. A comparison of two Chandra HETG observations obtained during the 2005 outburst of GRO J1655-40. Miller et al. (2006, 2008) and Kallman et al. (2009) analysed the April (red) spectrum, which was taken during an unusual "hypersoft" state. By contrast, the parameters adopted in our simulations are more representative of the soft-intermediate state during which the March (black) spectrum was obtained.

PYTHON, the heating and cooling rates for each cell are imported into ZEUS and these rates are compared to the heating and cooling rates used in the last hydrodynamic cycle. The normalization factors are then adjusted to make the rates to be used in the next cycle equal to those seen in the PYTHON simulation.

In order to avoid discontinuous changes in the heating and cooling rates, we apply a numerical damping factor during the recalibration after each IRT step. Since we expect and find - that our converged simulation reaches a steady state, the exact way in which the simulation approaches this state is not critical. This numerical damping therefore helps with convergence, but does not affect the final results. We have carried out several tests to verify this, using different damping factors, for example. Our final damping factor for these runs is 0.9 - meaning that the calibration factors can only change by about $10 \%$ after each IRT step.

Our simple implementation of RHD is computationally expensive. The computational effort is totally dominated by the IRT steps, each of which takes about $50 \times$ longer than a single HD step, even with the IRT calculations running on 256 processors in parallel. It is therefore not (yet) feasible to follow every HD step with a new IRT step. In order to overcome this problem, we inspect the properties of the outflow after each HD step and call PYTHON if more than $10 \%$ of the cells have changed their density by more than $50 \%$, or if $t_{H D, \text { max }}=1000 \mathrm{~s}$ of simulation time have elapsed since then. Again, this treatment is reasonable, because our thermally driven disc winds tend to a steady state. We consider a run to be converged if the mass-loss rate is no longer changing significantly. We have verified that our value of $t_{H D \text {, } \max }$ is reasonable by carrying out test calculations with different $t_{H D, \text { max }}$ in a small simulation domain (meaning that the run can be converged in a reasonable time, but at the cost of no longer capturing the Mach surface). In these test runs, reducing $t_{H D, \text { max }}$ by an order of magnitude increases the mass-loss rate by about $30 \%$.

\subsection{Heating and cooling rates}

Our modified versions of the approximate analytic heating and cooling rate equations are as follows. First, we split up the Compton heating $\left(H_{c}\right)$ and cooling $\left(\Lambda_{c}\right)$ rates into two separate terms. Thus Compton heating is described by

$H_{c}=K_{H_{c}} 8.9 \times 10^{-36} \xi T_{x}\left(\operatorname{ergs~s}{ }^{-1} \mathrm{~cm}^{3}\right)$,

while Compton cooling is modelled as

$\Lambda_{c}=K_{\Lambda_{c}} 8.9 \times 10^{-36} \xi(4 T)\left(\operatorname{ergs~s}^{-1} \mathrm{~cm}^{3}\right)$.

$\mathrm{T}$ is the electron temperature of the plasma, and the ionization parameter, $\xi$, is defined as

$\xi=\frac{L_{x}}{n_{H} r^{2}}$

where $L_{x}$ is the integrated luminosity above $13.6 \mathrm{eV}, n_{H}$ is the Hydrogen number density, and $\mathrm{r}$ is the distance between a given cell and the origin.

Splitting the heating and cooling rates allows us to calibrate the two terms independently, via their respective $K$-factors. This is necessary because Compton heating and cooling depend differently on the illuminating SED. With our new treatment of attenuation, different cells will generally see different SEDs, so our on-the-fly re-calibration needs to treat these two terms separately.

The so-called line cooling term $\left(\Lambda_{l}\right)$ includes contributions from both bound-bound and free-bound interactions. Our new version of this term is

$\Lambda_{l}=K_{\Lambda_{l}}\left(1.0 \times 10^{-16} \frac{\exp \left(-1.3 \times 10^{5} / T\right)}{T \sqrt{\xi}}+\mathcal{K}(T)\right)$ 
The units are the same as for Equation 4 and

$$
\mathcal{K}(T)=\left\{\begin{array}{lr}
5.0 \times 10^{-27} T^{1 / 2} & T<10^{4} \mathrm{~K} \\
1.0 \times 10^{-24} & 10^{4} \mathrm{~K}<T<10^{7} \mathrm{~K} \\
1.5 \times 10^{-17} T^{-1} & T>10^{7} \mathrm{~K} .
\end{array}\right.
$$

Blondin's (1994) original formulation corresponds to the intermediate temperature regime in Equation 6. However, in our simulations, we sometimes encounter dense regions near the mid-plane where the gas is no longer fully ionized. The low-temperature regime in Equation 6 accounts for this onset of partial ionization, where the cooling rate starts to depend on the temperature-sensitive collisional ionization rate. Similarly the high-temperature regime in Equation 6 accounts for the roll-off in the recombination cooling rate when the gas becomes over-ionized. This actually has little impact, since the cooling rate at such high temperatures is dominated by Compton cooling. It should be noted that this modified formula on its own does not represent a better fit to line cooling rates over all ionization parameters and temperatures. It is, however, a much better fit at low ionization parameters and allows us to achieve excellent agreement with the IRT rates (in combination with the $K_{\Lambda_{l}}$ calibration factor).

The "X-ray" (photo ionization + Auger) heating rate $\left(H_{x}\right)$ and bremsstrahlung cooling rate $\left(\Lambda_{b}\right)$ equations are unchanged from Blondin (1994), except for the normalization factors. We nevertheless include them here for completeness:

$H_{x}=K_{H_{x}} 1.5 \times 10^{-21} \sqrt{\xi / T}\left(1-\left(T / T_{x}\right)\right)\left(\operatorname{ergs~s}^{-1} \mathrm{~cm}^{3}\right)$,

and

$\Lambda_{b}=K_{\Lambda_{b}} 3.3 \times 10^{-27} \sqrt{T}\left(\operatorname{ergs~s}{ }^{-1} \mathrm{~cm}^{3}\right)$.

All five heating and cooling rates are combined to give the total net heating rate

$\rho \mathcal{L}=n_{H}\left(n_{e} H_{c}+n_{H} H_{x}-n_{e} \Lambda_{c}-n_{e} \Lambda_{l}-n_{e} \Lambda_{b}\right)$.

This equation is somewhat modified from the form given in H17, because here we include the effect of partial ionization. Specifically, H17 used the multiplier of $n_{e} n_{H}$ for all heating rates, but the X-ray heating rate does not, in fact, depend on $n_{e}$. This is not significant for the optically thin HD simulations presented in $\mathrm{H} 17$, since $n_{e}=1.21 n_{H}$ for a fully ionized plasma with solar abundances. However, as noted above, and discussed in detail in Section 4, in our RHD simulations a dense region forms towards the mid-plane. This region is not fully ionized due to photo-absorption, and so $n_{e}$ can no longer be approximated there as a simple multiple of $n_{H}$. We have therefore carried out a set of stand-alone PYTHON simulations covering the critical range of temperatures and fit the resulting electron to Hydrogen density ratio with the following piecewise fit:

$$
\frac{n_{e}}{n_{H}}=\left\{\begin{array}{lr}
2.5 \times 10^{-52} T^{12.3} & T<15000 \mathrm{~K} \\
2.5 \times 10^{-3.8} T^{0.86} & 15000 \mathrm{~K}<T<33000 \mathrm{~K} \\
1.21 & T>33000 \mathrm{~K} .
\end{array}\right.
$$

The net heating rate given by Equation 9 enters into the equations of hydrodynamics via the energy equation,

$\rho \frac{D}{D t}\left(\frac{e}{\rho}\right)=-P \nabla \cdot v+\rho \mathcal{L}$

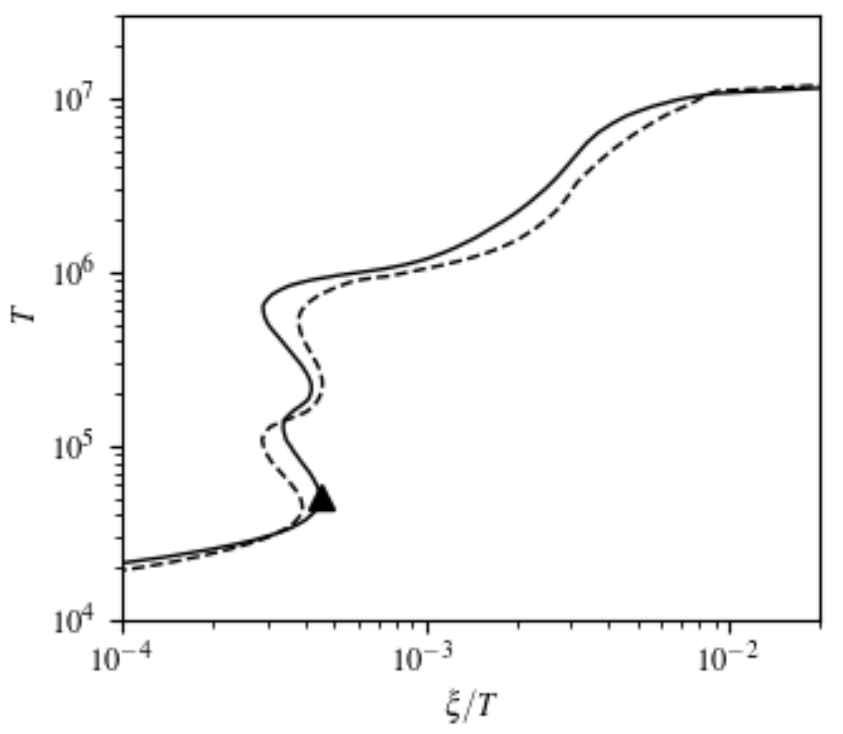

Figure 2. Stability plots generated using PYTHON (solid line) and CLOUDY (dashed line). The symbol highlights the location of the end of the lower temperature stable branch for the PYTHON simulation.

where $\rho$ is the mass density, $P$ is the pressure, $v$ is the velocity, $e$ is the internal energy and $\mathcal{L}$ is the net heating rate with units of ergs s${ }^{-1} \mathrm{~g}^{-1}$. The plasma is treated as an ideal monatomic gas, with $\gamma=5 / 3$.

\subsection{Boundary conditions and the computational grid}

The computational grid consists of one quadrant of a circle, with logarithmically spaced cells in the $r$ and $\theta$ direction. A reflecting boundary condition $(\mathrm{BC})$ is used along the mid-plane and an axisymmetric $\mathrm{BC}$ along the axis of rotational symmetry. Thus we are simulating a $2.5 \mathrm{D}$ spherical region centred on the accretor. The radial grid is finest towards the centre of the grid, with $d r_{k+1} / d r_{k}=1.02$. The polar grid runs from $0^{\circ}$ to $90^{\circ}$ in polar angle, with a spacing $d \theta_{k+1} / d \theta_{k}=0.95$. This gives more resolution towards the mid-plane, where we expect the density, velocity and temperature to change most quickly. In addition, we adopt outflow BCs at the inner and outer radial edges, and a minimum density of $10^{-22} \mathrm{~g} \mathrm{~cm}^{-3}$ is imposed throughout the grid. In the IRT simulations, we place a non-radiating, reflecting accretion disc at the mid-plane out to a radius of $2 R_{I C}$. This represents a reasonable maximum radius for a stable disk in this type of system (H17).

The density at the mid-plane of the grid out to a radius of $2 R_{I C}$ is also fixed and essentially acts as a mass reservoir for the simulation. This density has to be high enough to ensure that the simulation domain encompasses the critical acceleration zone of the outflow, but not so high as to include the entire body of the hydrostatic accretion disc. We satisfy these requirements by setting the mid-plane density on the basis of thermal stability considerations.

Figure 2 shows a set of thermal equilibrium curves generated from two different codes (PYTHON and CLOUDY) for 
different ways of calculating the heating and cooling rates. Each curve corresponds to an estimate of the equilibrium temperature as a function of $\xi / T$, i.e. the temperature for which heating equals cooling. The two codes give slightly different results, which is not unexpected since they use somewhat different atomic data, and the heating and cooling rates are very sensitive to exactly what processes and species are included.

In both calculations, we see that there is a low temperature branch at the left hand side, where the equilibrium temperature eventually drops to about $10000 \mathrm{~K}$. This gas can usefully be thought of as being part of the disc atmosphere. There is also a high temperature branch where the plasma reaches the Compton temperature. In between, the plasma becomes thermally unstable, sometimes being in a state when increasing the temperature actually increases the heating rate, allowing gas to heat up very rapidly. This is the region of interest for a thermal wind, since it is this heating that leads to expansion and therefore acceleration. Moreover, since we are interested in modelling the outflowing gas, rather than the static disc atmosphere, the mid-plane in our simulation would ideally correspond to the upper edge of the hydrostatic disc, i.e. to the hot end of the lower stable branch.

In the optically thin limit, we can achieve this by computing the critical ionization parameter, $\xi_{\text {cool,max }}$, at the end of this branch. We can then set the mid-plane density at all radii to the value necessary to give $\xi=\xi_{\text {cool, } \max }$. This is how we set the BC for the mid-plane density in H17. However, in the RHD simulations discussed here, we allow for the attenuation of the radiation field incident on a given grid cell by all of the intervening material along the line of sight to the central source. We also include electron scattering, which can be important in allowing otherwise shadowed parts of the wind to be illuminated indirectly (Sim et al. 2010; Higginbottom et al. 2014).

As a result of these two effects, the physical properties of the gas and the radiation field are coupled, and it is not possible to calculate $\xi_{\text {cool, max }}$ and the corresponding midplane density a priori. We can nevertheless safely adopt the mid-plane density estimated for optically thin conditions, since attenuation by intervening material can only reduce the strength of the incident radiation field and the associated ionization parameter. Setting the mid-plane density in this way therefore guarantees that $\xi<\xi_{\text {cool,max }}$. This is at the expense of including at least some material from further down on the lower stable branch in the simulation, i.e. material that belongs to the hydrostatic disc.

For the heating and cooling rates calculated with PYTHON in Figure 2, we find $\xi_{\text {cool, } \max }=22.7$ in the optically thin limit, with an equilibrium temperature $T_{\text {cool,max }}=$ $50000 \mathrm{~K}$ (marked with a triangle in Figure 2). The corresponding local mid-plane density is then calculated via

$\rho(r)=\rho_{0}\left(\frac{r}{R_{I C}}\right)^{-2}$,

with $\rho_{0}=1.6 \times 10^{-11} \mathrm{~g} \mathrm{~cm}^{-3}$. Here, $R_{I C}$ is the Compton radius, i.e. the radius at which the local isothermal sound speed (at the Compton temperature, $\mathrm{T}_{\mathrm{IC}}$ ) is equal to the escape velocity,

$R_{I C}=\frac{G M_{B H} \mu m_{H}}{k_{B} T_{I C}}$

\subsection{Summary of the simulations}

All of the key parameters that define our simulations and describe the resulting outflows are summarized in Table 1. Three different runs are listed in Table 1: (i) HD + CLOUDY $_{\tau \ll 1}-$ a pure hydrodynamic simulation in which radiative heating and cooling rates were calculated in the optically thin limit using CLOUDY; (ii) HD+PYTHON ${ }_{\mathcal{T} \ll 1}-$ a pure hydrodynamic simulation in which radiative heating and cooling rates were calculated in the optically thin limit using PYTHON; (iii) RHD - our new radiation-hydrodynamic simulation in which radiative heating and cooling rates were calculated accounting for attenuation (i.e. finite optical depth) in the wind using PYTHON.

The HD+CLOUDY $\tau \ll 1$ and HD+PYTHON ${ }_{\tau \ll 1}$ simulations are included as benchmarks for assessing the importance of radiative transfer effects in the RHD simulation. The HD + CLOUDY $_{\tau \ll 1}$ model is identical to the simulation shown in H17. The HD+PYTHON ${ }_{\tau \ll 1}$ calculation serves to confirm that, in the optically thin limit, we obtain very similar results when we use PYTHON instead of CLOUDY. Since the

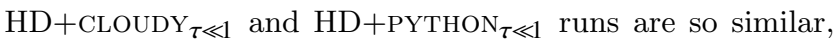
we will only plot and discuss the $\mathrm{HD}+\mathrm{PYTHON}_{\tau \ll 1}$ version below.

The third simulation in Table 1 is our new RHD calculation, where we iterate between HD and IRT steps. This allows us to relax the $\tau \ll 1$ assumption and to account for the attenuation of radiation field as it passes through the outflow.

\subsection{Limitation of the simulation: neglect of wind radiation.}

Perhaps the most significant approximation we still have to make is that the material in our simulation is optically thin to its own radiation. Thus while we calculate the full, frequency dependent spectrum produced by the gas in each grid cell self-consistently, we only use this to estimate the local radiative cooling. We do not consider the effect of cooling radiation produced in one grid cell on the thermal and ionization state of material in other grid cells.

This approximation is necessary because cells near the mid-plane in our simulations tend to become highly optically thick, a regime that PYTHON and most other Monte-Carlo based radiative transfer do not (yet) handle very well. The underlying problem is that, in order to capture the acceleration zone of the outflow, our simulation has to include at least some of the hydrostatic disc atmosphere. It is these deep-lying regions that cause numerical problems.

In order to check whether this approximation affects our results, we have carried out test calculations with PYTHON. Starting from a converged snapshot model, we excise the troublesome $\tau>>1$ disc regions, but then add a thin $T_{\text {eff }} \simeq 10^{4} \mathrm{~K}$ blackbody disc as a photon source "by hand". We find that the heating and cooling rates in the outflow (including the most important acceleration zone) following the addition of this extra source of photons do not change significantly. We therefore expect that the wind's own radiation field would not significantly affect the overall outflow properties. This expectation is supported by results from earlier 1D simulations which also showed that the inclusion of disk radiation did not significantly alter the structure or 
temperature balance of less dense material overlying it (Ko \& Kallman 1994).

\subsection{Comparing to observations: predicted absorption lines}

We generate synthetic absorption lines from our RHD simulation using a ray-tracing method similar to that described in H17. Briefly, we use a converged snapshot of the simulation to provide us with the density, velocity and ionization state throughout the outflow. For any given ground state transition, we can then calculate the optical depth as a function of velocity, $\tau(v)$, along any given sightline to the centre of the system. In doing so, we also account for the random thermal motions in the outflowing gas by adopting a Doppler-broadened Gaussian as the local line profile. The overall absorption line profile is then estimated as $e^{-\tau(v)}$.

The inclination towards GRO J1655-40 has been estimated to be $i=70.2^{\circ} \pm 1.9^{\circ}$ (Greene et al. 2001). However, as explained above, our simulations are designed to capture only the uppermost parts of the hydrostatic disc. If the disc itself is flared with a characteristic half-opening angle of $\gamma$, it would be more correct to adopt $i=70^{\circ}+\gamma$ in the spectral synthesis calculations. We will therefore show results for both $i=70^{\circ}$ and $i=80^{\circ}$ when comparing model spectra against observations.

\section{RESULTS}

Figure 3 shows a side-by-side comparison of the disc wind properties in a pure HD simulation $\left(\mathrm{HD}+\mathrm{PYTHON}_{\tau \ll 1}\right)$ versus those in our new RHD simulation. The upper panels of Figure 3 show the final (steady-state) density and velocity structure, while the lower panels show the corresponding thermal stability plots. Note that for the RHD simulation, the ionization parameter $\xi$ is calculated directly from the PYTHON IRT simulation, i.e. it is computed from the actual energy packets passing through each cell. Each point in these stability plots represents an individual grid cell, and the solid curves show the optically thin, PYTHON-based thermal equilibrium curve. As noted above, the key parameters of the outflows are summarized in Table 1.

A key feature in the new RHD simulation is a wedgeshaped, high-density region near the mid-plane (which extends out to $r=2 R_{I C}$ - the extent of our 'mass reservoir' BC). As discussed in Section 3.3, the mid-plane density we adopt guarantees that the RHD simulation grid covers the acceleration zone of the outflow, but it also means that this grid must include part of the hydrostatic disc. The formation of a dense, quasi-static region near the mid-plane in the RHD calculation is therefore inevitable. This structure broadly mirrors the 'disk' structure seen in 1D simulations (Raymond 1993; Ko \& Kallman 1994; Jimenez-Garate et al. 2002).

The wedge-like shape of this region can also be understood. X-rays emitted from the central source can only heat the atmosphere until the integrated optical depth along their path reaches $\tau \simeq 1$. For any given photon launch angle with respect to the mid-plane, there is thus a maximum radius out to which these photons can contribute to driving the outflow. Larger disc radii can only be reached by photons emitted with larger launch angles. The aspect ratio corresponding to the wind acceleration zone, $H / R$, must therefore increase with $R$, i.e. the shape of this zone must be (slightly) convex. At the outer edge of the hydrostatic disk $\left(r \simeq 10^{12} \mathrm{~cm}\right)$, the disk has an opening angle of about $4^{\circ}$. This is reasonably close to the disk opening angle of about $2.3^{\circ}$ inferred for the LMXB Cyg X-2 (Vrtilek et al. 1990)

Thermally stable grid cells within the body of the disc are represented by black symbols on the stability plot (bottom panels of Figure 3). They all have a temperature of around $12000 \mathrm{~K}$, with the radiative heating coming from the Auger ionization of metals. This process is associated with hard X-ray photons that are able to penetrate deep into the disc. The vertical density profile in the dense hydrostatic region is fairly flat, with cells maintaining roughly the same density as the mid-plane cell below. The boundary of the hydrostatic disc in the RHD simulation is very sharp, a phenomenon also seen in 1D simulations (Raymond 1993; Ko \& Kallman 1994; Jimenez-Garate et al. 2002). In those simulations, the transition between the cool 'disk' and the hotter coronal gas above it was unresolved. Unsurprisingly, we also do not resolve the transition, however changing the resolution at the transition region by increasing the number of cells in the $\theta$ direction has no effect on the overall structure of the resulting wind.

Above the disk, the temperature rapidly rises to a few million K. It is clear from the lower panels of Figure 3 that relatively few cells actually reach the stability curve, where radiative heating and cooling are in balance. This is because for the bulk of the simulation domain, adiabatic cooling is balancing radiative heating. Referring back to the $1 \mathrm{D}$ simulations of accretion disks, this means that most of the gas in the domain can be best described as an expanding disk atmosphere. This is exactly as one would expect, since we are concentrating on the outer parts of the disk where those simulations showed that, even neglecting adiabatic cooling, one only reaches the Compton temperature at a distance roughly equal to the launching radius. Another interesting observation is that the throughout the simulation domain, $\mathrm{X}$-ray heating and Bremsstrahlung cooling are important contributors to the balance; there are few locations where the temperature is set only by Compton effects

The density in the wind above this boundary is lower than in the optically thin HD run. This reduced density is reflected in a significantly $(\gtrsim 5 \times)$ reduced mass-loss rate of the RHD model compared to the pure HD simulations. However, even this reduced mass-loss rate amounts to more than twice the accretion rate onto the central object (as implied by our adopted luminosity). This 'wind efficiency' of about 2 is very close to that predicted by Done et al. (2018) for our disk size $\left(2 R_{I C}\right)$ and our luminosity $\left(0.5 L_{\text {crit }}\right.$, where $\left.L_{\text {crit }} \sim 0.03 T_{I C, 8}^{-0.5} L_{E d d}\right)$.

The terminal outflow velocity in the RHD model is comparable to that in the optically thin HD simulations, reaching $v_{\infty} \simeq 300 \mathrm{~km} \mathrm{~s}^{-1}$. This is in line with the expectation that $v_{\infty} \simeq v_{t h}$, since the characteristic temperature in outflowing material is $T \simeq 5 \times 10^{6} \mathrm{~K}$ (see Figure 3 ). At this temperature, the rms thermal speed of atoms is $v_{t h}=\sqrt{3 k_{B} T / \mu m_{p}} \simeq 450 \mathrm{~km} \mathrm{~s}^{-1}$, where $\mu \simeq 0.6$ is the mean molecular weight.

The dashed lines in the upper panels of Figure 3 show direction vectors corresponding to high-inclination lines of 


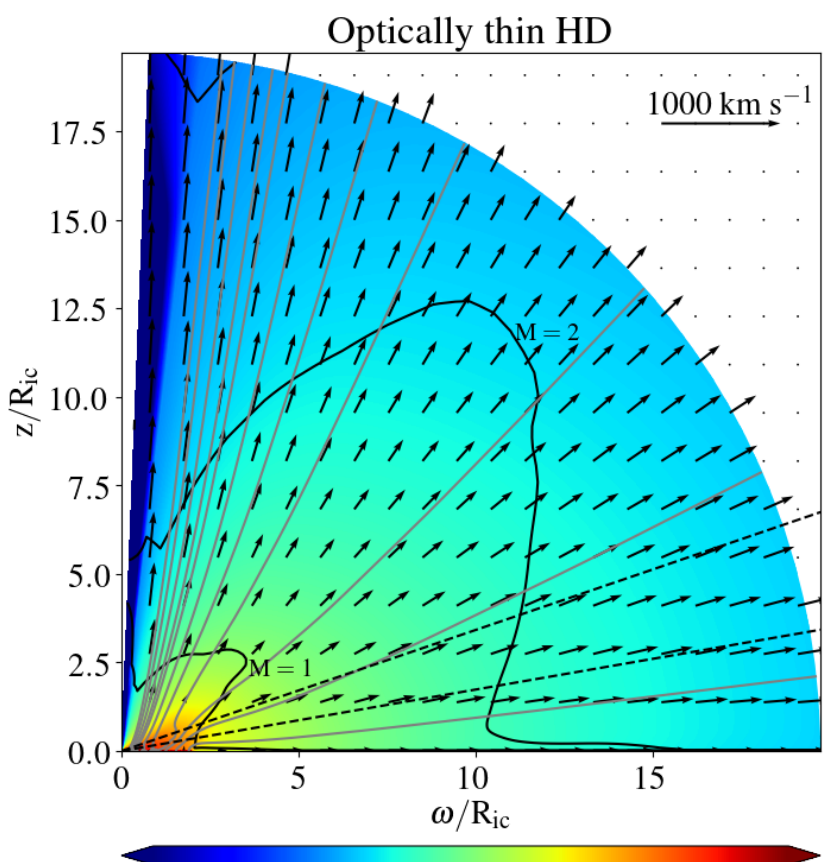

$\begin{array}{llllllll}-18.0 & -17.0 & -16.0 & -15.0 & -14.0 & -13.0 & -12.0 & -11.0\end{array}$ Density $\left(\mathrm{g} \mathrm{cm}^{-3}\right)$

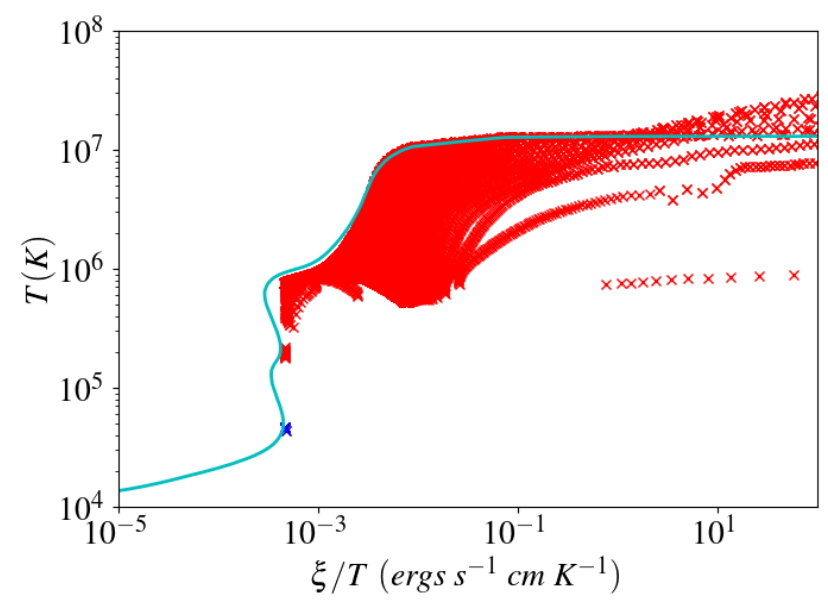

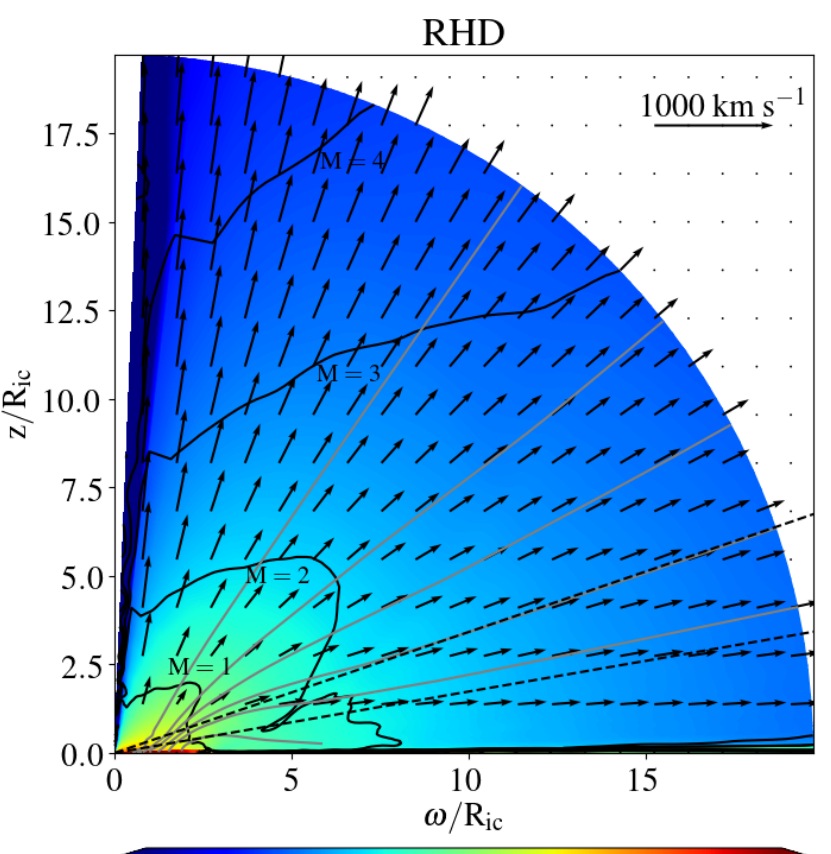

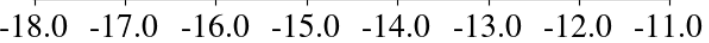
Density $\left(\mathrm{g} \mathrm{cm}^{-3}\right)$

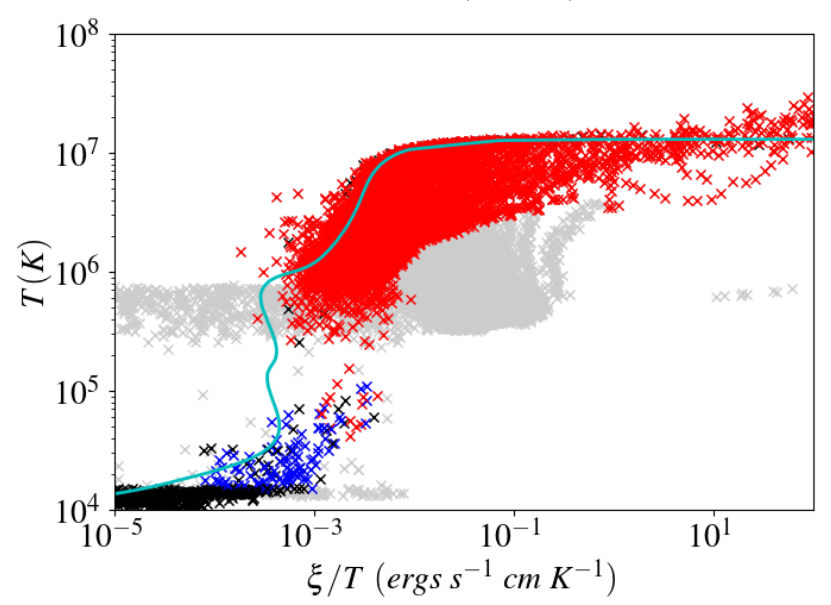

Figure 3. Upper panels: The density and velocity structure of the stable final state of the simulation. Grey lines show streamlines, and the black line shows the location of the Mach surfaces. The two dotted lines show the location of the $70^{\circ}$ and $80^{\circ}$ sightlines.

Lower panels: Stability plots for the same two snapshots. Blue symbols represents cells at the surface from which the wind is launched. Red symbols are for cells in the wind and black symbols are for cells below the wind-launch surface. The grey points in the right lower panel are for points outside the radial extent of the disc but at high inclination angle and so still shadowed by it.

sight with $i=70^{\circ}$ and $80^{\circ}$. They are provided here to highlight the inclinations for which we will show synthetic absorption line profiles in Section 5.2. These inclinations are typical of the soft-state LMXBs in which wind-formed absorption features have been observed (Ponti et al. 2012).

\section{DISCUSSION}

\subsection{The impact of attenuation}

As we have seen, our RHD simulation produce a disc wind with a roughly $5 \times$ lower mass-loss rate compared to optically thin, pure HD calculations. Thus while the pure HD calculations are useful for gaining qualitative insights into the nature of thermally-driven winds, radiative transfer effects are not negligible and must be taken into account in quantitative modelling efforts.

In order to illustrate how optical depth effects affect the properties of the resulting outflow, Figure 4 shows the radiation field seen in a set of typical cells in our RHD simulation. These curves show the mean intensity models computed by PYTHON for a set of cells near the acceleration zone of the outflow, i.e. straddling the upper surface of the disc-like structure towards the mid-plane.

These mean intensity models are calculated during the PYTHON cycles by logging each Monte Carlo energy packet which passes through (or is absorbed by) each cell in the model. These packets are initially generated over a wide con- 


\begin{tabular}{|c|c|c|c|}
\hline Simulation Method & HD & HD & RHD \\
\hline $\begin{array}{l}\text { Heating/Cooling } \\
\text { Rates }\end{array}$ & $\begin{array}{l}\text { CLOUDY } \\
\tau \ll 1\end{array}$ & $\begin{array}{l}\text { PYTHON } \\
\tau \ll 1 \\
\end{array}$ & $\begin{array}{l}\text { PYTHON } \\
\text { any } \tau\end{array}$ \\
\hline \multicolumn{4}{|l|}{ Physical Parameters } \\
\hline $\begin{array}{l}\mathrm{M}_{\mathrm{BH}}\left(\mathrm{M}_{\odot}\right) \\
\mathrm{T}_{\mathrm{x}}\left(10^{7} \mathrm{~K}\right) \\
\mathrm{L}_{\mathrm{x}}\left(10^{37} \mathrm{ergs} \mathrm{s}^{-1}\right) \\
\log \left(\xi_{\text {cold,max }}\right) \\
\mathrm{T}_{\mathrm{eq}}\left(\xi_{\text {cold, } \max }\right)\left(10^{3} \mathrm{~K}\right) \\
\rho_{0}\left(10^{-12} \mathrm{~g} \mathrm{~cm}^{-3}\right) \\
\mathrm{R}_{\mathrm{IC}}\left(10^{11} \mathrm{~cm}\right)\end{array}$ & $\begin{array}{l}7 \\
5.6 \\
3.3 \\
1.226 \\
43.7 \\
20.3 \\
4.82\end{array}$ & $\begin{array}{l}7 \\
5.6 \\
3.3 \\
1.35 \\
50.7 \\
16.0 \\
4.82\end{array}$ & $\begin{array}{l}7 \\
5.6 \\
3.3 \\
- \\
50.7 \\
16.0 \\
4.82\end{array}$ \\
\hline \multicolumn{4}{|l|}{ Grid parameters } \\
\hline $\begin{array}{l}\mathrm{R}_{\min }\left(10^{10} \mathrm{~cm}\right) \\
\mathrm{R}_{\max }\left(10^{10} \mathrm{~cm}\right) \\
\mathrm{R}_{\text {disc }}\left(10^{10} \mathrm{~cm}\right) \\
\mathrm{R}_{\text {ratio }} \\
\mathrm{N}_{\mathrm{R}} \\
\theta_{\min } \\
\theta_{\max } \\
\theta_{\text {ratio }} \\
\mathrm{N}_{\theta}\end{array}$ & $\begin{array}{l}2.4 \\
960 \\
96 \\
1.05 \\
200 \\
0.0 \\
90.0 \\
0.95 \\
100\end{array}$ & $\begin{array}{l}2.4 \\
960 \\
96 \\
1.05 \\
200 \\
0.0 \\
90.0 \\
0.95 \\
100\end{array}$ & $\begin{array}{l}2.4 \\
960 \\
96 \\
1.05 \\
200 \\
0.0 \\
90.0 \\
0.95 \\
100\end{array}$ \\
\hline \multicolumn{4}{|l|}{ Derived wind properties } \\
\hline $\begin{array}{l}\mathrm{V}_{\mathrm{r}}\left(\rho>1 \times 10^{12}\right) \\
\max , \text { blueshifted }\left(\mathrm{km} \mathrm{s}^{-1}\right) \\
\mathrm{V}_{\mathrm{r}}\left(\max , \theta>60^{\circ}\left(\mathrm{km} \mathrm{s}^{-1}\right)\right. \\
\mathrm{N}_{\mathrm{H}}\left(70^{\circ}\right)\left(10^{22} \mathrm{~cm}^{-2}\right) \\
\mathrm{N}_{\mathrm{H}}\left(80^{\circ}\right)\left(10^{22} \mathrm{~cm}^{-2}\right) \\
\dot{\mathrm{M}}_{\text {wind, outer }}\left(10^{18} \mathrm{~g} \mathrm{~s}^{-1}\right) \\
\dot{\mathrm{M}}_{\text {wind, outer }}\left(\dot{\mathrm{M}}_{\mathrm{acc}}\right) \\
0.5 \mathrm{MV}_{\mathrm{r}}^{2}\left(10^{32} \mathrm{erg} \mathrm{s}^{-1}\right)\end{array}$ & $\begin{array}{l}19 \\
290 \\
13 \\
63 \\
6.7 \\
15 \\
24\end{array}$ & $\begin{array}{l}30 \\
280 \\
12 \\
55 \\
5.6 \\
13 \\
21\end{array}$ & $\begin{array}{l}47 \\
260 \\
2.0 \\
4.4 \\
1.1 \\
2.5 \\
4.2\end{array}$ \\
\hline
\end{tabular}

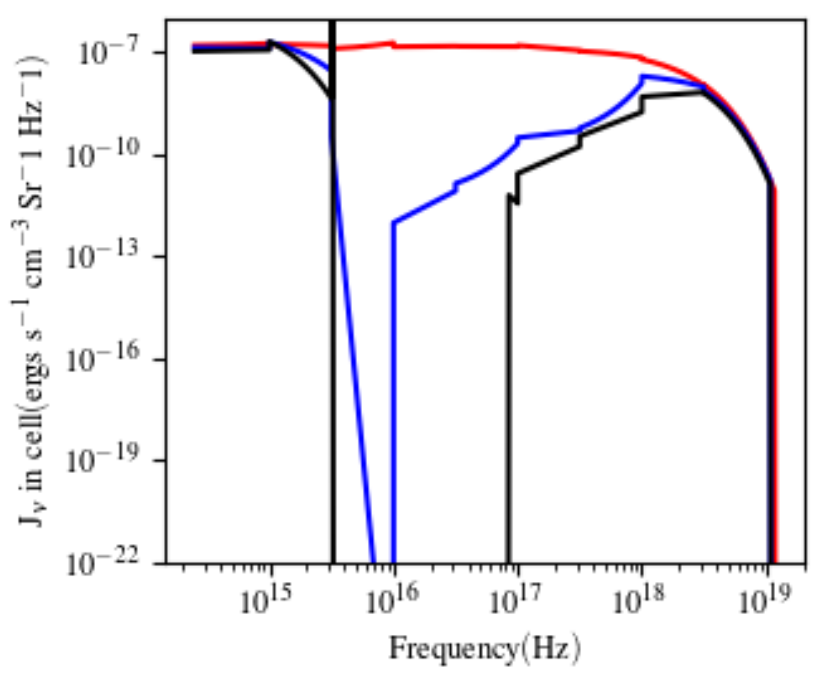

Figure 4. Mean intensity in a set of cells at $\omega=1.5 R_{I C}$. The blue curve is for a cell at the upper surface of the dense disc at the mid-plane. The red curve is for the lower density cell directly above it, and the black curve is for the disc cell directly below it.

Table 1. Parameters adopted in the simulations, along with key properties of the resulting outflows.

tinuous range of frequencies, but in individual cells the distribution is modelled in a series of frequency bins (two per frequency decade) using either a power law or exponential model. Deep absorption features are captured by truncating the frequency bins on the fly to take account of any frequencies where no packets are seen at all. This technique captures the gross features of the mean intensity very flexibly, and permits the calculation of photo-ionization rates for complex radiation fields reasonably accurately.

The blue curve in Figure 4 corresponds to a cell at the upper surface of the dense disc, while the red curve corresponds to the cell directly above it. The optical depth from the central object to this latter cell is small, so the red curve essentially shows the expected radiation field at this location in the absence of attenuation. This comparison clearly shows that the radiation field reaching the acceleration zone of the outflow is significantly attenuated, primarily due to photoionization of hydrogen. The result is a lower X-ray heating rate near the disc surface, and this is likely to be the main reason for the lower mass-loss rate seen in the RHD simulation.

The black curve in Figure 4 is typical of the radiation field in cells lying deeper within the disc. At these depths, there are essentially no hydrogen ionizing photons left, so this dense part of disc is relatively cool and mostly neutral in

the RHD simulation. The dominant ionization and heating process in these cells is due to inner shell photo-ionization of metals by X-ray photons. This provides enough heating to keep the temperature at around $12,000 \mathrm{~K}$ - the temperature of the cool stable branch in the bottom right panel of Figure 3.

Figure 4 shows that the radiation field in the disc/wind transition region can be strongly modified by absorption along the line of sight. This in turn reduces the ionization rate in this region, and hence reduces the local heating and cooling rates (predominantly photo-ionization heating and recombination/line cooling). In light of Figure 4, the significant differences in the physical outflow properties seen in our HD and RHD simulations should not come as a surprise.

Previous simulations of disk-winds have demonstrated that indirect illumination by scattered radiation can also be important (Sim et al. 2010; Higginbottom et al. 2014). In our RHD simulation, the ionizing photon field in cells in the disc, with its high column densities towards the central source, is indeed dominated by radiation scattered from electrons in the wind back down towards the disc. At the boundary between the disc and the wind, this effect is smaller, with at most $30 \%$ of the ionizing photons in these cells arriving indirectly. Indirect irradiation is therefore a secondary effect in this particular simulation, but the ability to include it in our RHD calculations is an important advance.

\section{$5.2 \quad$ Predicted line profiles}

Figure 5 shows the intrinsic profile of the wind-formed Fe XXV $1.85 \AA$ absorption line in our simulation, i.e. before taking any instrumental resolution into account. This shows that there is significant optical depth out to roughly $v_{\infty} \simeq 300 \mathrm{~km} \mathrm{~s}^{-1}$. The positive velocity tail of the profile reaching $\simeq+100 \mathrm{~km} \mathrm{~s}^{-1}-$ is due to the significant thermal Doppler broadening in the outflowing gas.

Figure 5 also illustrates that line strength is a clear func- 


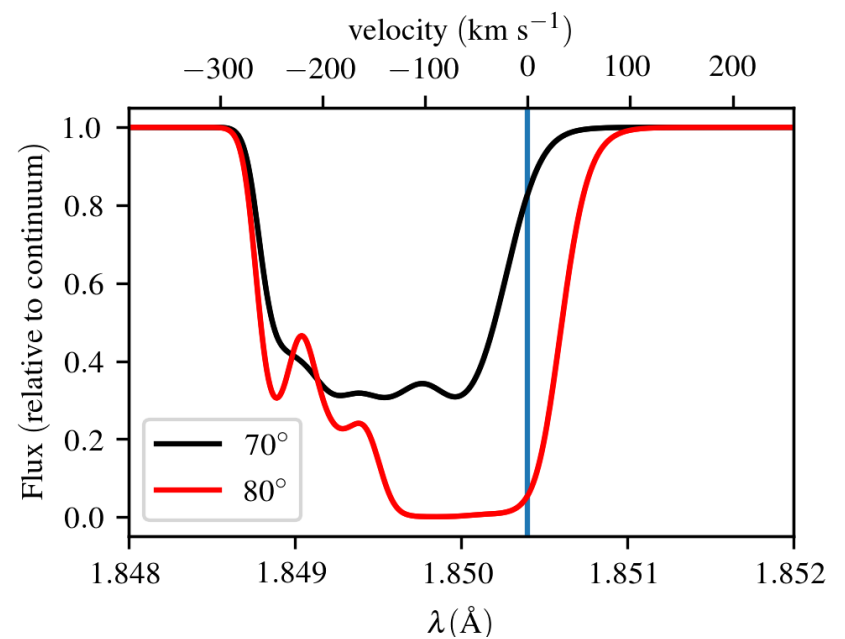

Figure 5. Predicted absorption line profiles for the Fe xxv Lyman $\alpha$ transition at $1.8504 \AA$, as viewed from $i=70^{\circ}$ and $i=80^{\circ}$.

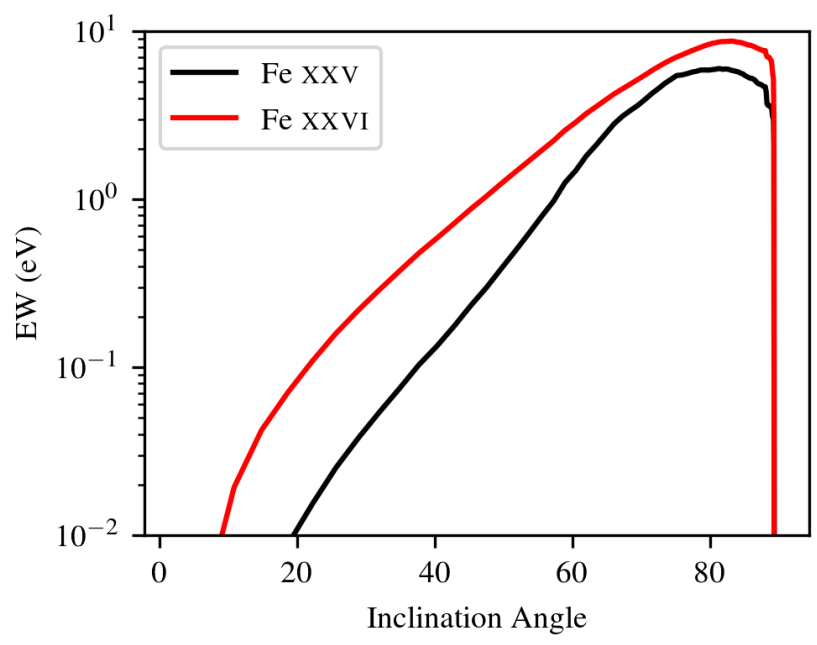

Figure 6. Absorption line equivalent width associated with Fe XXV $1.85 \AA$ and the Fe XXvi $1.78 \AA$ doublet.

tion of inclination, with higher-inclination sightlines producing stronger lines than lower-inclination ones. This trend is a natural consequence of the equatorial nature of the outflow, since higher (column) densities are preferentially found near the disc plane (see Figure 3). We illustrate this more directly in Figure 6, which shows the dependence of the predicted Fe Xxv $1.85 \AA$ and Fe xxvi $1.78 \AA$ equivalent widths on inclination. Observable features with $\mathrm{EW} \gtrsim 1 \mathrm{eV}$ are only predicted for inclinations $i \gtrsim 60^{\circ}$. This is comparable to the range of inclinations for which wind-formed absorption lines have been observed (Ponti et al. 2012).

\subsection{Comparison to Observations}

As discussed in Section 2, the parameters adopted in our RHD simulations roughly match those of GRO J1655-40 in its soft-intermediate state. Are the Fe Xxv and Fe XXVI absorption lines we predict consistent with the CHANDRA HETG observations obtained during this state?

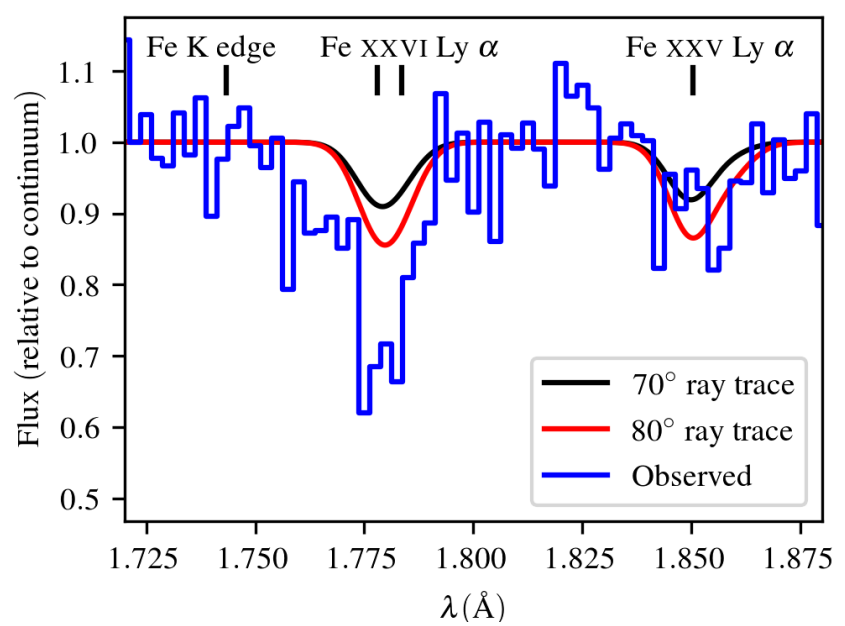

Figure 7. $80^{\circ}$ 'ray-traced' spectrum around the ground state Fe XXV and XXVI resonance lines (grey line). The red line is the effect of convolving this spectrum with a $0.012 \AA$ FWHM Gaussian to represent the resolution of the HETG and the blue line is the March 2006 Chandra observation of GRO J1655-40. This is scaled to a continuum based on the flux at $1.7 \AA$ and $1.9 \AA$ to allow comparison with the ray-traced spectrum.

Figure 7 shows a direct comparison between predicted and observation absorption line profiles. Here, we have convolved the predicted profiles with the CHANDRA HEG resolution of $0.012 \AA($ FWHM $)$, corresponding to $\Delta v \simeq 2000 \mathrm{~km} \mathrm{~s}^{-1}$ at $1.8 \AA$. Even though the lines are weak, the data are noisy, and the profiles are unresolved, it is encouraging that the simulations predict line strengths and line ratios that are within a factor of two or so of the observed ones. In assessing the quality of this agreement, it is important to remember that Figure 7 is not a fit to the observations. No parameters were adjusted to optimize the match to the data. Given all of the uncertainties and approximations associated with such a comparison, this level of agreement is promising.

Lee et al. (2002) and Ponti et al. (2012) have used the wind-formed Fe XXV and Fe XXVI absorption lines in LMXBs to estimate the mass-loss rates in these outflows. Using our synthetic line profiles, we can test how well this method recovers the true mass-loss rate in the RHD simulation. Briefly, the method is based on combining the mass continuity condition,

$\dot{M}_{\text {wind }}=\Omega R^{2} n m_{p} v_{\text {wind }}$,

with the definition of the ionization parameter, $\xi$, given in Equation 4. The result is

$\dot{M}_{\text {wind }}=\Omega m_{p} v_{\text {wind }} L_{x} / \xi$.

Here, $\Omega$ is the solid angle subtended by the outflow, and $m_{p}$ is the proton mass.

Observationally, $L_{x}$ is determined from the X-ray continuum and $v_{\text {wind }}$ from the intrinsic width and/or blueshift of the absorption line. The solid angle of an equatorial outflow along the disc plane with half-opening angle $\alpha$ is just $\Omega=4 \pi \sin \alpha$. As noted above, the range of inclinations for which wind-formed absorption lines have been observed suggests $\alpha \simeq 30^{\circ}$ (Ponti et al. 2012), in line with what we find 


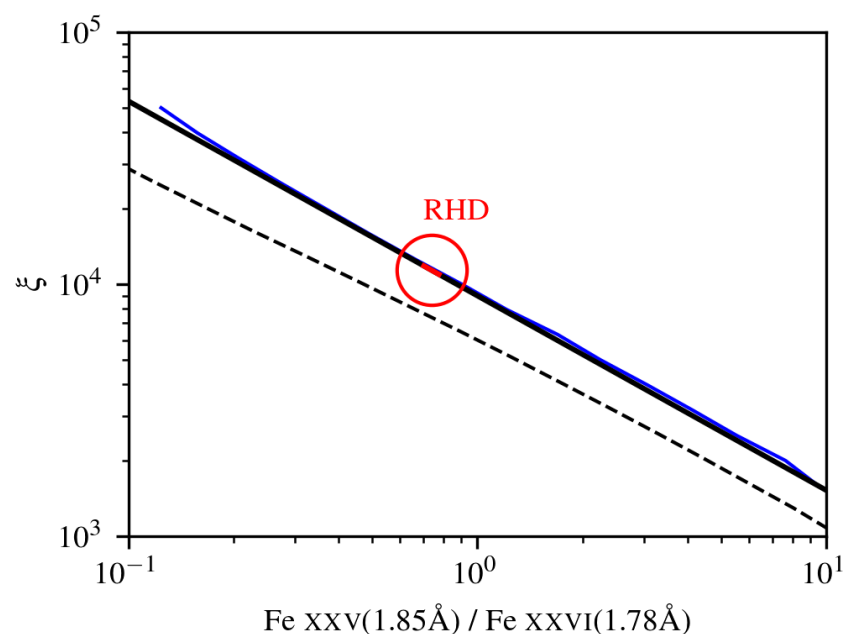

Figure 8. Relationship between ratio of Fexxv and Fexxvi Lyman $\alpha$ equivalent widths and ionization parameter - based on data from Kallman \& Bautista (2001). Dashed line shows relationship based on data from PYTHON simulations using the same bremsstrahlung SED as used in the RHD simulation

in our RHD simulation. The solid angle subtended by the outflow is therefore roughly $\Omega \simeq 2 \pi$.

This leaves only the ionization parameter, $\xi$, to be estimated. This is done by measuring the Fe xxv / Fe xxvI line ratio and comparing it to single-cloud, optically thin ionization calculations for a particular reference SED (e.g. Kallman \& Bautista 2001). The dependence of ionization parameter on the Fe line ratio in the calculations of Kallman \& Bautista (2001) is shown by the solid line on Figure 8. For the line ratios we obtain from our synthetic spectra for sightlines in the range $70^{\circ} \leq i \leq 80^{\circ}$ (about 0.7-0.8), we would estimate $\xi \simeq 1.1 \times 10^{4}$ for the line-forming region in our outflow.

As noted by Ponti et al. (2012), the actual SED does, of course, have a significant impact on the line ratios. For reference, we therefore also show in Figure 8 the relationship between the Fe line ratio and ionization parameter as calculated with PYTHON for the actual (unattenuated) SED used in our RHD simulation. This relationship would give an estimate of $\xi \simeq 7 \times 10^{3}$.

Adopting $L_{x}=3.3 \times 10^{37} \mathrm{erg} \mathrm{s}^{-1}, v_{\text {wind }} \simeq 300 \mathrm{~km} \mathrm{~s}^{-1}$, $\Omega \simeq 2 \pi$ and $\xi \simeq 10^{4}$ as characteristic numbers for the wind in the RHD simulation, we obtain an "empirical" estimate of $\dot{M}_{\text {wind }} \simeq 10^{18} \mathrm{~g} \mathrm{~s}^{-1}$. This is reassuringly close to the actual mass-loss rate through the boundary of our computational grid, $\dot{M}_{\text {wind }}=1.1 \times 10^{18} \mathrm{~g} \mathrm{~s}^{-1}$, and demonstrates the robustness of this method of measuring mass-loss rates.

With this in mind, how close is the mass-loss rate in our model to the observationally inferred one for the softintermediate state? Ponti et al. (2012) estimated luminosities and mass-loss rates from a set of Chandra HETG observations of black-hole LMXBs, including GRO J1655-40. We reproduce their results in Figure 9, highlighting the two measurements they obtained for GRO J1655-40. These correspond to the two Chandra HEG spectra shown in Figure 1, i.e. they represent the March 2005 soft-intermediate state and the April 2005 hypersoft state. Remarkably, the mass-loss rate we obtain from our RHD simulation is a close match to the rate inferred for the soft-intermediate state of GRO J1655-40.

It should be noted that at $80^{\circ}$, the lines are saturated. In this case the velocity spread of material contributing to the absorption is more important to the line EW than the mass-loss rate. In addition, the Fe XXV inter-combinational line at $1.8595 \AA$ will be blended with the resonance line. This line is normally very weak in comparison but if the resonance line is saturated, it could measurably change the EW of the blend. These two effects could slightly alter the line ratios and hence massloss rate.

\section{SUMMARY}

High inclination LMXBs show clear evidence of disc winds when they are in disc-dominated soft states. In order to test whether these winds may be thermally driven, we have developed a new operator-splitting method for carrying out radiation-hydrodynamic simulations of these outflows, based on combining elements of the hydro-code ZEUS and the radiative transfer code PYTHON. The resulting RHD code allows for arbitrary SEDs and accounts for full frequencydependent radiation transfer and ionization calculations.

We then carried out RHD simulations of a thermallydriven wind in an LMXB, using the same basic system parameters $\left(M_{B H}, \mathrm{~L}_{X}, \mathrm{SED}\right.$, etc.) as used in earlier simulations that assumed that outflow to be optically thin throughout.

Our main conclusions are as follows:

- In terms of its geometry and velocity field, the outflow that develops in our RHD simulation is qualitatively similar to the winds seen in pure HD simulations that adopt the optically thin limit. However, the mass-loss rate is much lower, $\dot{M}_{w} \simeq 1 \times 10^{18} \mathrm{~g} \mathrm{~s}^{-1}$ in the RHD simulations, compared to $\dot{M}_{w} \simeq 6 \times 10^{18} \mathrm{~g} \mathrm{~s}^{-1}$ in the optically thin HD calculation. Nevertheless, this reduced mass-loss rate still represents approximately $2.5 \times$ the accretion rate.

- The reason for this reduced mass-loss rate is strong photo-absorption between the X-ray source and the windlaunching region in the model. This decreases the amount of heating in these cells. Thus properly incorporating radiative transfer into simulations of thermally-driven disc winds is important.

- The system parameters of our fiducial model broadly match those of the well-known LMXB system GRO J165540. The mass-loss rate in our RHD simulation is similar to that estimated by Ponti et al. (2012) for GRO J1655-40 in a soft-intermediate state. Moreover, ray-traced absorption line profiles generated for our simulated outflow show reasonable agreement with a Chandra spectrum obtained from this source in the soft-intermediate state.

Overall, our results suggest the that thermal driving remains a viable mechanisms for powering the disc wind in (most) LMXBs.

\section{ACKNOWLEDGEMENTS}

Calculations in this work made use of the Iridis4 Supercomputer at the University of Southampton. NH and CK acknowledge support by the Science and Technology Facilities 


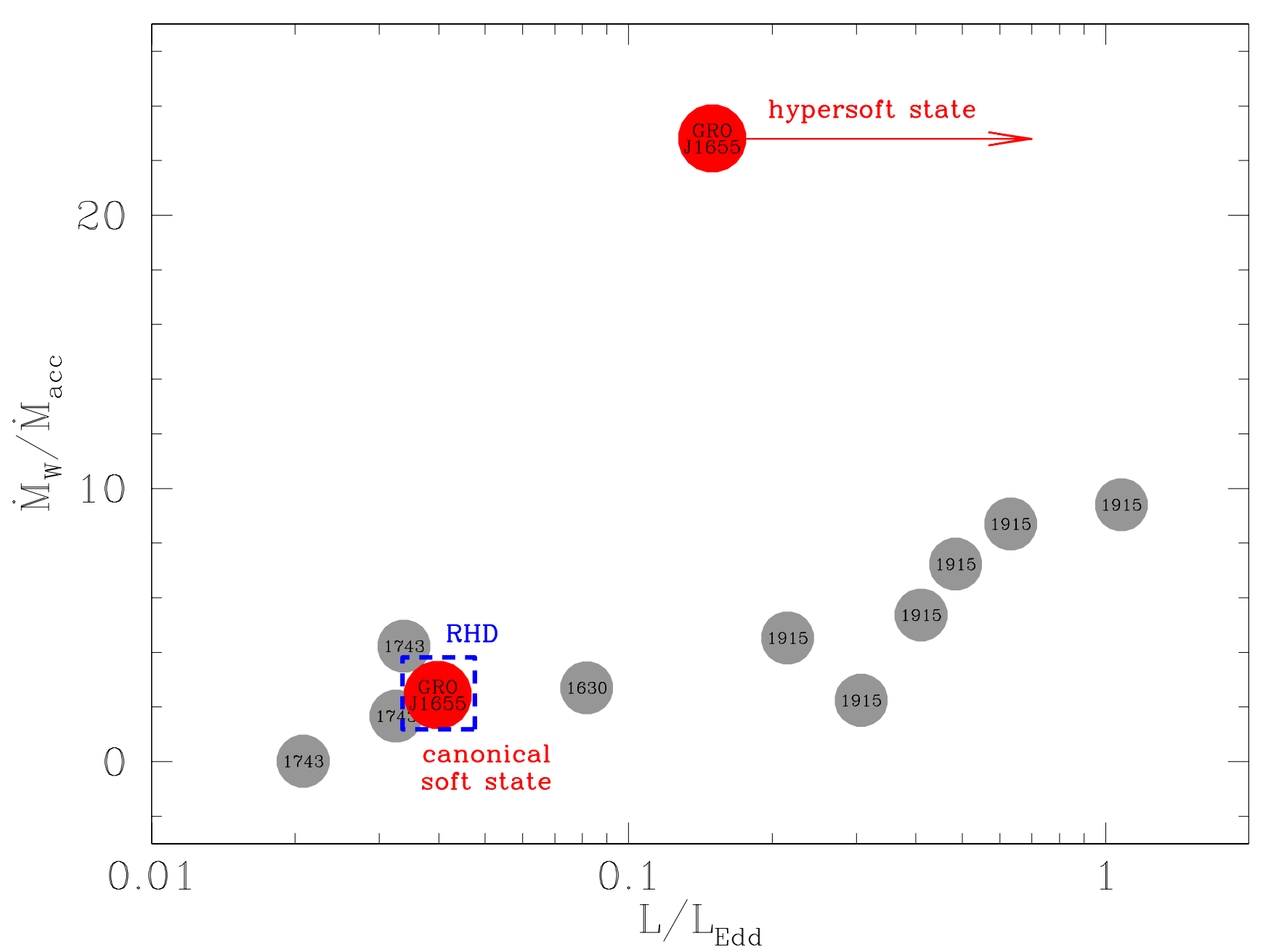

Figure 9. $\dot{M}_{\text {wind }} / \dot{M}_{a c c}$ vs. luminosity based upon figure 5 in Ponti et al. (2012). Filled circles are empirical values obtained from Chandra HETG data for several LMXBs. The labels inside the circles refer to the specific object each measurement refers to $(1743=$ H1743-322; $1630=4 \mathrm{U}$ 1640-47; $1915=$ GRS 1915+105; GRO J1655 = GRO J1655-40). The two red circles represent the measurements obtained for GRO J1655-40 in the hypersoft and soft-intermediate states. As discussed in Section 2, the measurement for the hypersoft state might be a lower limit (if the outflow in this state was Compton-thick). The green square shows the ratio of mass-loss rate to accretion rate predicted by an optically thin, pure HD simulation. The blue square shows the same ratio for our RHD simulation, demonstrating the excellent agreement with the empirical result for GRO J1655-40 in the soft-intermediate state.

Council grant ST/M001326/1. KSL acknowledges the support of NASA for this work through grant NNG15PP48P to serve as a science adviser to the Astro- $\mathrm{H}$ project, JHM is supported by STFC grant ST/N000919/1 and SAS is supported by STFC through grant, ST/P000312/1. Finally we thank the referee John Raymond for a very useful review which suggested many interesting comparisons.

\section{REFERENCES}

Begelman M. C., McKee C. F., Shields G. A., 1983, ApJ, 271, 70 Belloni T. M., 2010, in Belloni T., ed., Lecture Notes in Physics, Berlin Springer Verlag Vol. 794, The Jet Paradigm: from Micro- quasars to Quasars. p. 53

Blandford R. D., Payne D. G., 1982, MNRAS, 199, 883

Blondin J. M., 1994, ApJ, 435, 756
Chakravorty S., et al., 2016, A\&A, 589, A119

Cordova F. A., Mason K. O., 1982, ApJ, 260, 716

Díaz Trigo M., Boirin L., 2013, Acta Polytechnica, 53, 659

Díaz Trigo M., Boirin L., 2016, Astronomische Nachrichten, 337, 368

Done C., Tomaru R., Takahashi T., 2018, MNRAS, 473, 838

Dyda S., Dannen R., Waters T., Proga D., 2017, MNRAS, 467, 4161

Emmering R. T., Blandford R. D., Shlosman I., 1992, ApJ, 385, 460

Fabian A. C., 2012, ARA\&A, 50, 455

Frank J., King A. R., Lasota J.-P., 1987, A\&A, 178, 137

Greene J., Bailyn C. D., Orosz J. A., 2001, ApJ, 554, 1290

Higginbottom N., Proga D., 2015, ApJ, 807, 107

Higginbottom N., Knigge C., Long K. S., Sim S. A., Matthews J. H., 2013, MNRAS, 436, 1390

Higginbottom N., Proga D., Knigge C., Long K. S., Matthews J. H., Sim S. A., 2014, ApJ, 789, 19 
Higginbottom N., Proga D., Knigge C., Long K. S., 2017, ApJ, 836,42

Icke V., 1980, AJ, 85, 329

Jimenez-Garate M. A., Raymond J. C., Liedahl D. A., 2002, ApJ, 581,1297

Kallman T., Bautista M., 2001, ApJS, 133, 221

Kallman T. R., Bautista M. A., Goriely S., Mendoza C., Miller J. M., Palmeri P., Quinet P., Raymond J., 2009, ApJ, 701, 865

Knigge C., Long K. S., Blair W. P., Wade R. A., 1997, ApJ, 476, 291

Ko Y.-K., Kallman T. R., 1994, ApJ, 431, 273

Konigl A., Kartje J. F., 1994, ApJ, 434, 446

Körding E. G., Knigge C., Tzioumis T., Fender R., 2011, MNRAS, 418, L129

Lee J. C., Reynolds C. S., Remillard R., Schulz N. S., Blackman E. G., Fabian A. C., 2002, ApJ, 567, 1102

Long K. S., Knigge C., 2002, ApJ, 579, 725

Luketic S., Proga D., Kallman T. R., Raymond J. C., Miller J. M., 2010, ApJ, 719, 515

Matthews J. H., Knigge C., Long K. S., Sim S. A., Higginbottom N., 2015, MNRAS, 450, 3331

Miller J. M., Raymond J., Fabian A., Steeghs D., Homan J., Reynolds C., van der Klis M., Wijnands R., 2006, Nature, 441, 953

Miller J. M., Raymond J., Reynolds C. S., Fabian A. C., Kallman T. R., Homan J., 2008, ApJ, 680, 1359

Mirabel I. F., Rodríguez L. F., 1999, ARA\&A, 37, 409

Neilsen J., Homan J., 2012, ApJ, 750, 27

Neilsen J., Rahoui F., Homan J., Buxton M., 2016, ApJ, 822, 20

Netzer H., 2006, ApJ, 652, L117

Ponti G., Fender R. P., Begelman M. C., Dunn R. J. H., Neilsen J., Coriat M., 2012, MNRAS, 422, 11

Proga D., Kallman T. R., 2002, ApJ, 565, 455

Proga D., Stone J. M., Kallman T. R., 2000, ApJ, 543, 686

Raymond J. C., 1993, ApJ, 412, 267

Remillard R. A., McClintock J. E., 2006, ARA\&A, 44, 49

Shidatsu M., Done C., Ueda Y., 2016, ApJ, 823, 159

Shields G. A., McKee C. F., Lin D. N. C., Begelman M. C., 1986, ApJ, 306, 90

Shlosman I., Vitello P., 1993, ApJ, 409, 372

Shlosman I., Vitello P. A., Shaviv G., 1985, ApJ, 294, 96

Sim S. A., Miller L., Long K. S., Turner T. J., Reeves J. N., 2010, MNRAS, 404, 1369

Stone J. M., Norman M. L., 1992, ApJS, 80, 753

Turner T. J., Miller L., 2009, A\&ARv, 17, 47

Uttley P., Klein-Wolt M., 2015, MNRAS, 451, 475

Vrtilek S. D., Raymond J. C., Garcia M. R., Verbunt F., Hasinger G., Kurster M., 1990, A\&A, 235, 162

Weymann R. J., Morris S. L., Foltz C. B., Hewett P. C., 1991, ApJ, 373, 23

Woods D. T., Klein R. I., Castor J. I., McKee C. F., Bell J. B., 1996, ApJ, 461, 767

Zensus J. A., 1997, ARA\&A, 35, 607

()

This paper has been typeset from a $\mathrm{T}_{\mathrm{E}} \mathrm{X} / \mathrm{LAT}_{\mathrm{E}} \mathrm{X}$ file prepared by the author. 